\title{
M1 macrophage-derived exosomes moderate the differentiation of bone marrow mesenchymal stem cells
}

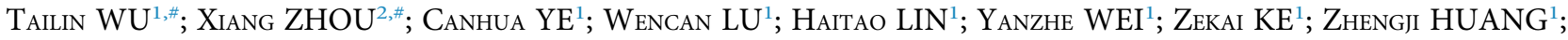 \\ Jianzhou LUO $^{1}$; Huiren TAO ${ }^{1}$; Chunguang DUAN ${ }^{1, *}$ \\ ${ }^{1}$ Shenzhen University General Hospital, Shenzhen, 518055, China \\ ${ }^{2}$ Luoyang Central Hospital Affiliated to Zhengzhou University, Luoyang, 417000, China
}

Key words: M1 macrophages, Exosomes, Osteogenesis, RUNX2

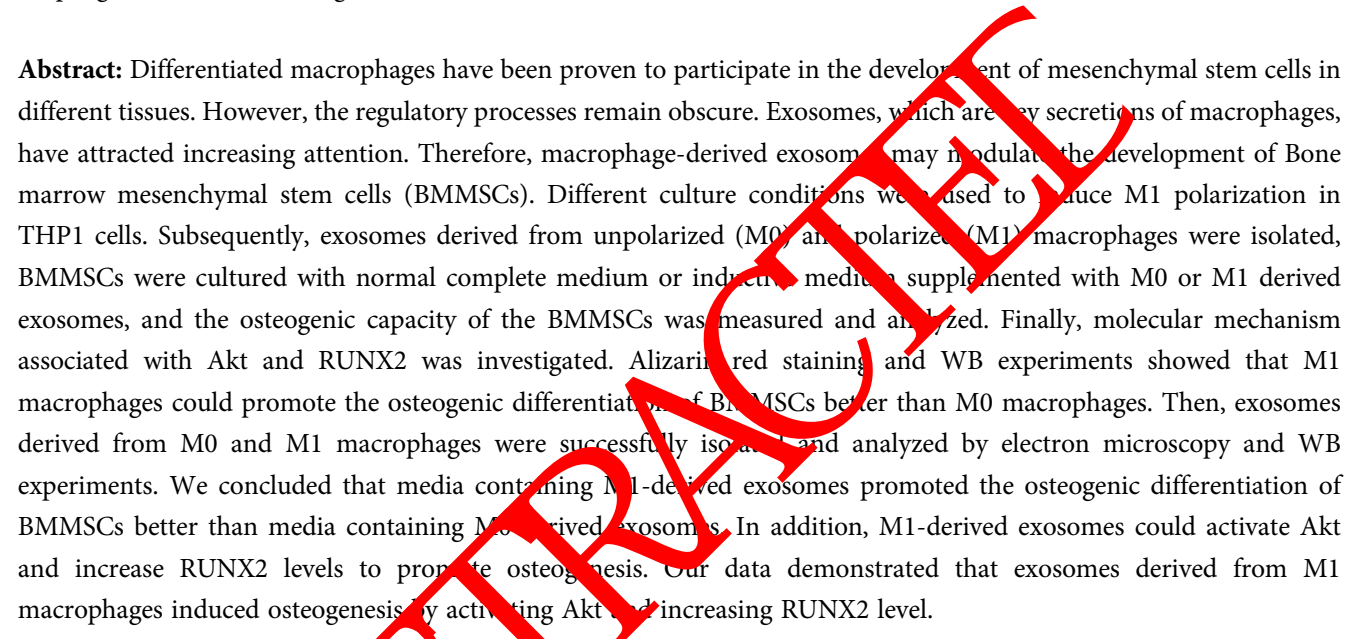

\section{Introduction}

During the previous time, stem cen herapy has become the focus of regenerative medicine and th engineering (Wei et al., 2013). BMMSCs, due to their advantages, stand out from a variety of stem cells and are the most promising option for allogeneic and autologous transplantation (Hu et al., 2018; Ma et al., 2018). However, the clinical application of BMMSCs is associated with is associated with many challenges, such as low dose, inefficiency and poor survival rates (Regmi et al., 2019). Considerable efforts have improved the regeneration of mesenchymal stem cells (Fahy et al., 2014). At present, the importance in regulation of macrophages in the BMMSCs has been widely recognized (Pajarinen et al., 2019; Zhang et al., 2017b).

\footnotetext{
*Address correspondence to: Chunguang Duan, duanchunguangdor@163.com

${ }^{\#}$ These authors contributed equally to this work

Received: 01 December 2020; Accepted: 22 April 2021
}

Macrophages, as important components of the innate immune system, were recently discovered to participate in tissue regeneration ( $\mathrm{Yu}$ et al., 2016). Under various stimuli, macrophages can change from an unpolarized (M0) to a differentiated state (M1 or M2) and play significant roles in many stages of tissue healing. M1 macrophages generally participate in wound inflammation and play a proinflammatory role. M2 macrophages, on the other hand, have anti-inflammatory functions and induce tissue healing (Mcdonald et al., 2014; Zhang et al., 2017b). In the past, numerous reports have demonstrated the regulatory effects of macrophages on mesenchymal stem cells (Maxson et al., 2012; Pajarinen et al., 2019; Sesia et al., 2015; Zhang et al., 2017a).

Exosomes are single-membrane vesicles that have the same topology as the cell, and diameters of $\sim 30$ to $\sim 200 \mathrm{~nm}$, and are important regulators of critical physiological and pathological processes (Kalluri and Lebleu, 2020; Lee et al., 2019). Exosomes take up nucleic acids, lipids, proteins and other substances, which can be released into the extracellular environment and internalized by target cells, subsequently regulating cellular physiological and 
pathological processes (Zhang et al., 2019). In fact, there is increasing evidence suggesting that exosomes released from macrophages are important regulators of many biological activities (Li et al., 2019; Poltavtseva et al., 2019; Wei et al., 2019). However, the underlying mechanism of macrophagederived exosomes with different phenotypes in regulating BMMSCs is still unclear.

RUNX2 is an important transcriptional regulator of osteogenesis (Chava et al., 2018). RUNX2 deficient mice fail to develop bone cells (Thacker et al., 2016). The differentiation experiment demonstrates that RUNX2 is an important transcription factor in bone development. RUNX2 has important regulatory effects on the differentiation of osteoblasts, the maturation of chondrocytes, the differentiation of osteoclasts, and the secretion of extracellular matrix (Chava et al., 2018; Kim et al., 2018).

RUNX2 is primarily used for mesenchymal cell commitment to osteoblastic cell lines, which are necessary for the development of immature osteoblasts but not for the further maturation of osteoclasts (Omar et al., 2011). This finding indicates that RUNX2 is essential for osteogenesis. However, this difference is mainly due to differences in the cellular environment, as glucose appears to induce bone maturation by regulating the synthesis of Type I collagen (Ikebuchi et al., 2018; Omar et al., 2011). This finding suggests that specific regulatory signals are requires for the different commitment processes.

Based on the published works, we hypothesized that exosomes were the key mediators of macrophage BMMSCs. We also further elucidate the role of ey ou from type macrophages in the differentiation of MMS The results are prospected to improve und stan in or interaction between macrophages and $\mathrm{BMM}$ and ha to better regulate the effectiveness of mese hyma tem ceils in tissue regeneration.

\section{Materials and Methods \\ Isolation and culture of BMMSCs}

Approximately female C57BL/6 mica aged 5 weeks were purchased from the Experimental Animal Center of the Shenzhen University and were sacrificed and the femurs and tibias dissected. After two washes with PBS, bone marrow cells were flushed from the bones into $10-\mathrm{cm}$-culture dishes using MEM (Invitrogen) supplemented with 10\% FBS (Invitrogen) and $1 \%$ penicillin and streptomycin (Sigma Aldrich). Then, the dishes were incubated at $37^{\circ} \mathrm{C}, 5 \% \mathrm{CO}_{2}$ incubator. Cells from the 2 nd or 3 rd passage were used in the analysis.

Characterization of BMMSCs and macrophages by flow cytometry

To analyze the expression of surface markers characteristic of BMMSCs or and macrophages, FACS was performed using specific fluorochrome-conjugated monoclonal antibodies corresponding to each cell type. Briefly, $1 \times 106$ cells were washed with $10 \% \mathrm{FBS} / \mathrm{PBS}$ and centrifuged at $300 \times \mathrm{g}$ for $5 \mathrm{~min}$ at room temperature to pellet the cells. Subsequently, primary antibodies purchased from Abcam, anti-CD90 (ab225), anti-CD29 (ab183666), anti-CD34 (ab81289),
anti-CD45 (ab10558), anti-CD63 (ab213090), and anti-iNOS (ab115819) at a concentration of $2 \mathrm{mg} / \mathrm{mL}$ for the identification of BMMSCs or macrophages. Subsequently, unbound antibody was removed by washing with $2 \mathrm{~mL}$ of $10 \% \mathrm{FBS} / \mathrm{PBS}$, and pellets were resuspended in $500 \mu \mathrm{L}$ PBS and examined by flow cytometry, with 10,000 events recorded for each condition. Flow cytometry data was analyzed using BD CellQuest ${ }^{\mathrm{TM}}$ Pro software Version 5.1 (BD Bioscience).

THP-1 cell culture, macrophage differentiation and polarization

Human leukemia monocytic THP-1 cells were purchased from Procell (Cat. CL-0233, Wuhan, China). THP-1 cells were maintained in RPMI 1640 (Gibco) supplemented with $10 \%$ Fetal Bovine Serum (FBS) and $2 \mathrm{mM}$ L-glutamine. THP-1 monocytes (Mo) were differentiated into resting macrophages (M0) using $100 \mathrm{nM}$ phorbol 12-myristate 13acetate (PMA, Solarbio, P6741) for $72 \mathrm{~h}$ followed by $24 \mathrm{~h}$ in PMA-free medium (PMA-resting, PMAr). For M1 polarization $\mathrm{M} 0 \mathrm{~m}$. crophages were further cultured in M1polarization meliu containing $100 \mathrm{ng} / \mathrm{mL}$ LPS (SigmaAldrich) and $20 \mathrm{ng} / \mathrm{m}$ int feron gamma (IFN $\gamma$, Solarbio, P00041)

Oil red staining

The BMMS induction for 18 days, cells were washed once ith PBS, fixea by treatment with $4 \%$ paraformaldehyde for 3. min at oom temperature, and then stained with Oil Red som on (Jiancheng Biotechnology, Nanjing, China) for Onin at room temperature. A microscope was used to observe the stained cells.

\section{ALP staining}

BMMSCs were seeded into 24 -well plates at a density $1 \times 104$ cells $/ \mathrm{cm}^{2}$ until $70 \%$ confluence was reached. Then, the old complete medium was removed, and fresh medium containing $0.1 \mu \mathrm{mol} / \mathrm{L}$ dexamethasone (Cat\# RASMX-90021, Oricell, Cyagen Biosciences) was added to the plates. All groups were cultured for 3 days prior to ALP staining. The cells were washed thrice with PBS and fixed by treatment with 4\% Paraformaldehyde Fix Solution (Cat\# E672002, Sangon Biotech, Shanghai, China) for $1 \mathrm{~min}$. Then, the cells were incubated with ALP for $20 \mathrm{~min}$ at $25^{\circ} \mathrm{C}$ in the dark and observed under a light microscope.

\section{Isolation and identification of exosomes}

After the cells reached $80 \%$ confluence, serum-free culture medium was added, and the supernatants were collected after culturing for $24 \mathrm{~h}$. Then, the exosomes were isolated from the supernatants through traditional ultracentrifugation: $2,000 \times$ $\mathrm{g}$ for $30 \mathrm{~min}$ to remove the cells and debris, centrifugation at $10,000 \times \mathrm{g}$ for $30 \mathrm{~min}$ to remove the subcellular components, and centrifugation at $100,000 \times \mathrm{g}$ for $70 \mathrm{~min}$ to obtain the exosomes. Finally, the exosomes were resuspended in $0.01 \mathrm{M}$ PBS, centrifuged at $100,000 \times \mathrm{g}$ for $70 \mathrm{~min}$ for purification, and preserved in a freezer at $-80^{\circ} \mathrm{C}$. A Zeta View system was used to measure the exosome concentration and size distribution, and transmission electron microscopy was used to detect exosome morphology. The exosome surface markers were analyzed by Western blotting. 


\section{Exosome treatment}

Exosomes were isolated from $5 \times 106 \mathrm{M} 0$ and M1, BMMSCs were planted into 6-well plates one day before treatment. When the cells grew at about $70 \%$ of confluent, $200 \mu \mathrm{g}$ of exosomes were directly added into cells. Two days after treatment, cells were collected for the following experiments.

RNA extraction and quantitative real-time PCR

Total cellular RNA was isolated using a single-step method with TRIzol (Invitrogen) according to the manufacturer's instructions. First-strand complementary cDNA was synthesized from $1 \mu \mathrm{g}$ of total RNA according to the manufacturer's instructions. The reaction was terminated by heating at $70^{\circ} \mathrm{C}$ for $5 \mathrm{~min}$. The single-strand cDNA was quantified by spectrophotometer so as to use $10 \mathrm{ng}$ of cDNA in each Real-Time PCR well.

Quantitative real-time PCR was performed using the Applied Biosystems StepOne ${ }^{\mathrm{TM}}$ Real-Time PCR System and the Fast $\mathrm{SYBR}^{\circledR}$ Green Master Mix reagent. The quantification of gene expression for each target gene and reference gene was performed in separate tubes. The relative expression level of the target gene was normalized to that of the endogenous reference GAPDH gene and the $2-\Delta \Delta \mathrm{Ct}$ cycle threshold method was used to calculate the relative expression levels of the target genes defined by the primers.

ALP: Forward Primer 5' -ACCACCACGAGAGTGAACCA-3', Reverse Primer 5'-CGTTGTCTGAGTACCAGTCCC-3'; Osterix: Forward Primer 5'-CCTCTGCGG GACTCAACAAC-3'Reverse Primer 5'-AGCCCATTAGTd CTTGTAAAGG-3'; Runx2: Forward Primer 5'-TG CTGTCATGGCGGGTA-3', Reverse Primer 5'-2 'TCA ;ATCGTTGAACCTTGCTA-3'; AKT: Fory $1 \mathrm{~d}$, mer 5'-AGCGACGT GGCTATTGTGAAG-3', Re ce Primo 5'GCCATCATTCTTGAGGAGGAAGT-3'; BMP Primer 5'-ACCCGCTGTCTTCTAGC 1-3', Revers Primer 5'- TTTCAGGCCGAACATGCTGAC

\section{Immunoblotting analysis}

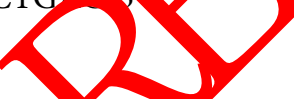

The protein samples were isolated om BMMSCs by using lysis buffer (P0013G, Beyotime, Shan hai, China). Protein lysates were quantified using Bradford protein assay kit (ab102535, Abcam, Cambridge, UK), and then $20 \mu \mathrm{g}$ protein was performed electrophoresis on SDS-PAGE (10\%; Beyotime, Shanghai, China) and then transferred on PVDF membranes (Millipore, Billerica, MA, USA). Subsequently, the PVDF membranes were incubation with ALP (1:1000, ab229126, Abcam, Cambridge, UK), Osterix (1:1000, ab229258, Abcam, Cambridge, UK) and Runx2 (1:1000, ab76956, Abcam, Cambridge, UK), CD9 (1:500, ab263024, Abcam, Cambridge, UK), CD63 (1:500, ab134045, Abcam, Cambridge, UK), AKT (1:1000, ab8805, Abcam, Cambridge, UK), p-AKT (1:1000, ab8933, Abcam, Cambridge, UK), BMP2 (1:1000, ab14933, Abcam, Cambridge, UK) and GAPDH (1:5000, ab8245, Abcam, Cambridge, UK) at $4^{\circ} \mathrm{C}$ overnight. Then it was incubated with secondary antibody by using HRP goat antirabbit/mouse IgG antibodies (1:20000, ab8245, Abcam, Cambridge, UK). Enhanced chemiluminescence detection system (Millipore, Massachusetts, USA) was used for photographing of the protein peptides on the PVDF membranes.

\section{Alizarin Red S staining}

Cells were seeded into 24 -well plates at a density of $1 \times 104 \mathrm{cells} / \mathrm{cm}^{2}$ overnight. The following day (Day 0), media were changed to DMEM, 10\% FBS, $1 \%$ penicillin-streptomycin, $50 \mu \mathrm{g} / \mathrm{mL}$ ascorbic acid, $10 \mathrm{mM} \beta$-glycerophosphate (Control media) or DMEM, $10 \%$ FBS, $1 \%$ penicillin-streptomycin, $50 \mu \mathrm{g} / \mathrm{mL}$ ascorbic acid, $10 \mathrm{mM} \beta$-glycerophosphate, $100 \mathrm{ng} / \mathrm{mL}$ BMP-2 (Osteogenic media). Media were changed every $72 \mathrm{~h}$. After 27 days, cells were washed with PBS, fixed with $10 \%$ neutral buffered formalin for $30 \mathrm{~min}$, stained with $2 \%$ Alizarin Red stain for $45 \mathrm{~min}$, rinsed three times with distilled water, air-dried and imaged using an inverted microscope equipped with a color camera.

\section{BMMSCs transmission electron microscopy}

The exosomes were immediately fixed with $4 \%$ glutaraldehyde for $2 \mathrm{~h}$ at $4^{\circ} \mathrm{C}$ after isolation, washed 3 times with $0.1 \mathrm{~mol} / \mathrm{L}$ PBS, fixed with $1 \%$ osmium tetroxide for $2 \mathrm{~h}$, and dehydrated with conventional ethanol and acetone. Exosomes was impremnated with epoxy resin, embedded, and polymerized. ter that, $0.5-\mu \mathrm{m}$-thick semi-thin sections were prepared no the ultri-thin sections of $60 \mathrm{~nm}$ were prepared aft localis tion of the light microscope. The sections wer tai ed wit uranium dioxide acetate and lead citrate rd visut ized ader an electron microscope.

\section{atisncal an ysis}

Il statisti 1 lanalyses were performed with SPSS 19.0 (Chicago, softwa) or GraphPad Prism 8 software (San Diego, CA, SAy. . cesults were repeated at least three times and are now as mean \pm SEM. We combined the quantitative data from three biological replicates and analyzed the results with Statistical significance between groups was determined by Student's $t$-test or one-way analysis of variance (ANOVA). $P<0.05$ were considered statistically significant.

\section{Results}

Effects of macrophages on the differentiation of BMMSCs To examine the effect of macrophages on osteogenic differentiation, we first induced THP1 cells to differentiate into functional macrophages. After treatment with different cytokines, cellular morphology was examined. THP1 cells treated with LPS and IFN- $\gamma$ displayed higher cell numbers than the control and PMA treated groups, suggesting that the proliferation of macrophages was enhanced (Fig. 1A). In addition, flow cytometry was performed to analyze the cell surface markers on macrophages. Compared to control and PMA treated THP1 cells, LPS and IFN- $\gamma$ treated macrophages showed significant increase in CD68 and iNOS (specific markers of M1 macrophages) (Fig. 1B). These results suggested that THP1 cells were successfully induced to undergo M1 polarization by LPS and IFN- $\gamma$.

Subsequently, to investigate the effect of differentiated macrophages, including the M0 and M1 types, on the osteogenic differentiation abilities of BMMSCs, BMMSCs were cocultured with different types of differentiated macrophages. After 14 days of culture, ALP activity analysis was performed to analyze the extent of osteogenesis as shown in Suppl. Fig. 1. Quantitative analysis revealed that M1 macrophages but not M0 macrophages increased the ALP activity of BMMSCs in 
A

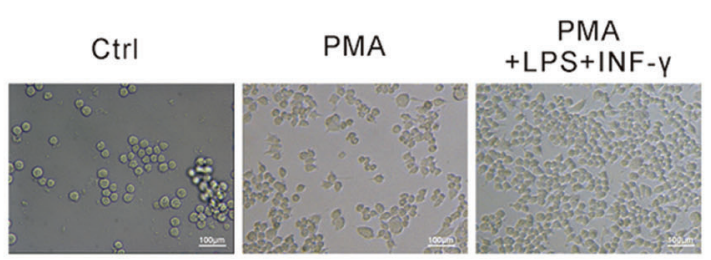

C

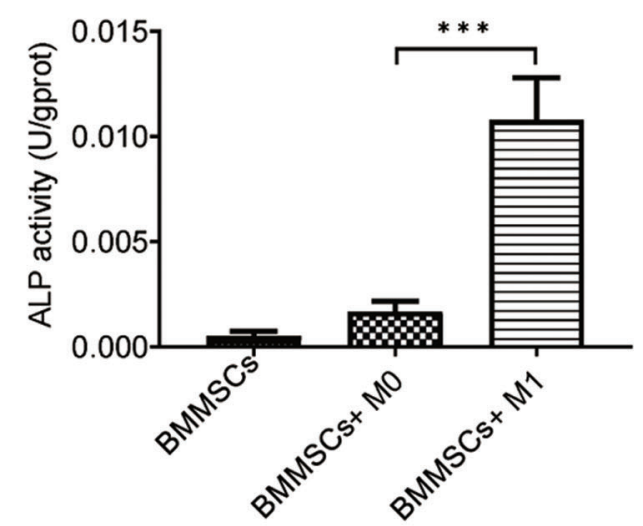

B
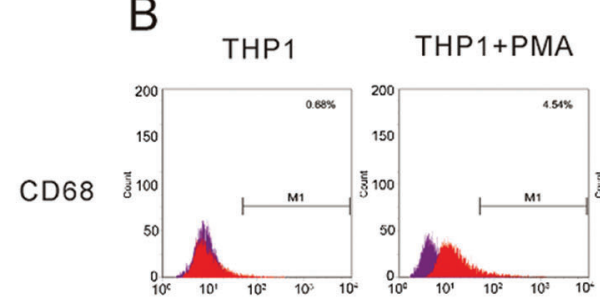

THP1+PMA + LPS + INF-Y
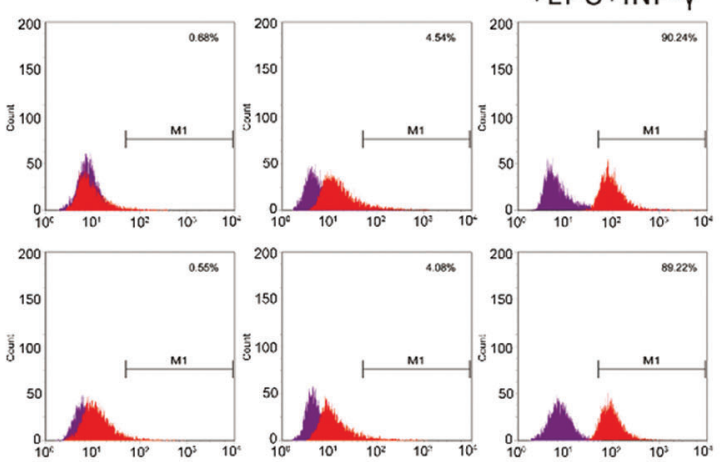

E

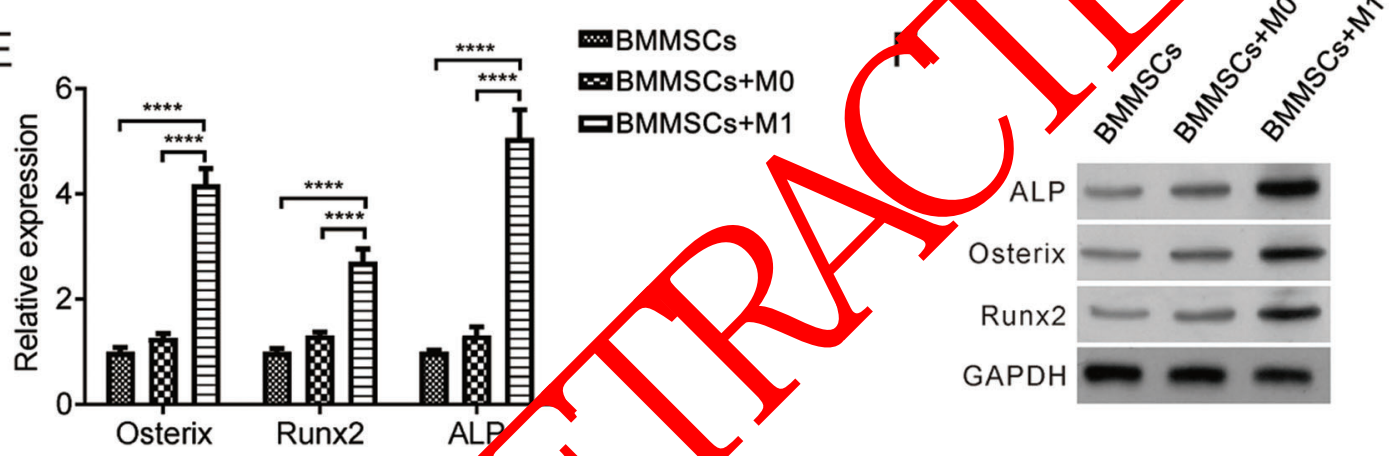

Alizarin red staining

\begin{tabular}{ccc}
\multicolumn{3}{c}{ Alizarin red staining } \\
\hline BMMSCs & BMMSCs+M0 & BMMSCs+M1
\end{tabular}

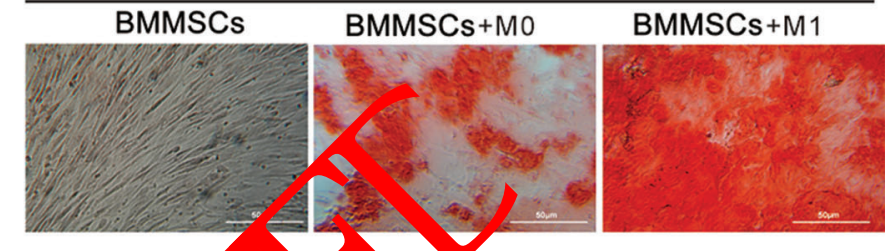
FIGURE 1. Effects of macrophages on the steog dic differntiation of BMMSCs. (A) Representative images of THP1cells after culture in
various conditions. (B-C) Flow cytomerns in the indicated treated or unstimulated cells. (D) Alizarin red S staining of ells u der iff ent culture conditions. (E-F) Expression levels of osteogenesis-related genes (ALP, Osterix and Runx2) in BMMSCs. ${ }^{\star}$ s.0u ${ }^{\star} P<0.001$ vs. BMMSCs; Ctrl, control; M0 unpolarized macrophages; M1, polarized macrophages; PMA, phorbol 12-myristar 13-acetate.

the coculture system. This result was further confirmed by Alizarin red $S$ staining. The results showed that BMMSCs cultured with M1 macrophages formed the highest number of positively stained cells. In contrast, M0 macrophages showed little influence on the development of BMMSCs (Fig. 1D). In addition, the mRNA and protein levels of osteogenesis related genes were analyzed after cells were subjected to osteogenic induction for 7 days. The data showed that BMMSCs cocultured with M1 macrophages had significantly increased mRNA and protein levels of ALP, Osterix and Runx2 (Figs. $1 \mathrm{E}$ and $1 \mathrm{~F})$. These results suggested that M1 macrophages could promote the osteogenesis of BMMSCs.

Characterization of different macrophage-derived exosomes It has been reported that exosomes participate in the osteogenesis of BMMSCs (Ekstrom et al., 2013; Wei et al., 2019). Toinvestigate the underlying mechanisms of macrophages affect the osteogenesis of BMMSCs, we focused our attention on exosomes derived from macrophages. First, exosomes were isolated from M0 or M1 macrophages. Transmission electron microscopy (TEM) analysis showed that the isolated exosomes were small round nanometer sized particles with bilayer membranes (Fig. 2A). Moreover, markers of exosomes, such as CD9 and CD63, were all expressed on the M0 and M1-derived exosomes (Fig. 2B). These results ensured the successful isolation of exosomes from M0 and M1 macrophages.

Effects of macrophage-derived exosomes on the differentiation of the BMMSCs

To determine whether macrophage-derived exosomes affect the osteogenic differentiation of BMMSCs, an ALP activity assay was first conducted. The results revealed that ALP activity was dramatically increased by supplementation with M1-derived exosomes. However, M0 derived exosomes induced consistently low ALP activity (Fig. 3A). The effects of exosomes on BMMSC differentiation were also investigated by Alizarin red $\mathrm{S}$ staining. The results 
A

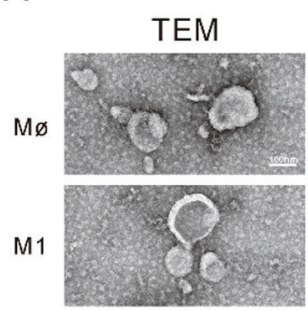

B

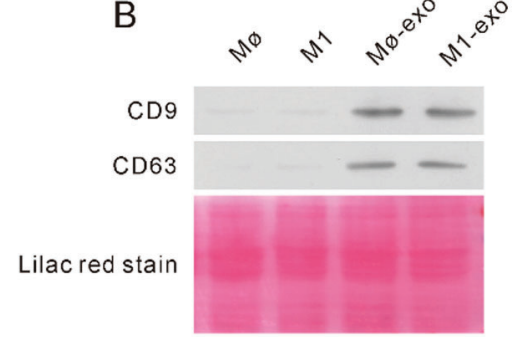

FIGURE 2. Characterization of different macrophage-derived exosomes (A) TEM analysis of M0 and M1-derived exosomes. TEM, Transmission Electron Microscope; M1, polarized macrophages; PMA, phorbol 12-myristate 13-acetate (B) The exosome marker (CD9 and CD63) in the M0 and M1-derived exosome.

showed that cells cultured with M1-derived exosomes had the highest number of positively stained cells, suggesting that these cells have the most robust differentiation potential. In contrast, cells cultured with M0-derived exosomes showed fewer positively stained cells, revealing low differentiation potential (Fig. 3B). In addition, the gene expression analysis of osteogenic differentiation-related genes, including Osterix, RUNX2 and ALP, further confirmed the difference in osteogenesis promotion in the $\mathrm{M} 0$ and $\mathrm{M} 1$ groups (Figs. 3C and 3D).

Osteogenesis is associated with M1-derived exosome-mediated activation of Akt and BMP2 in differentiation

Recent studies have revealed that the Akt signaling pathwa plays a role in BMMSC differentiation. Moreove Sh re BMP2 has been confirmed to be an important sc strate of osteoblast differentiation.

A

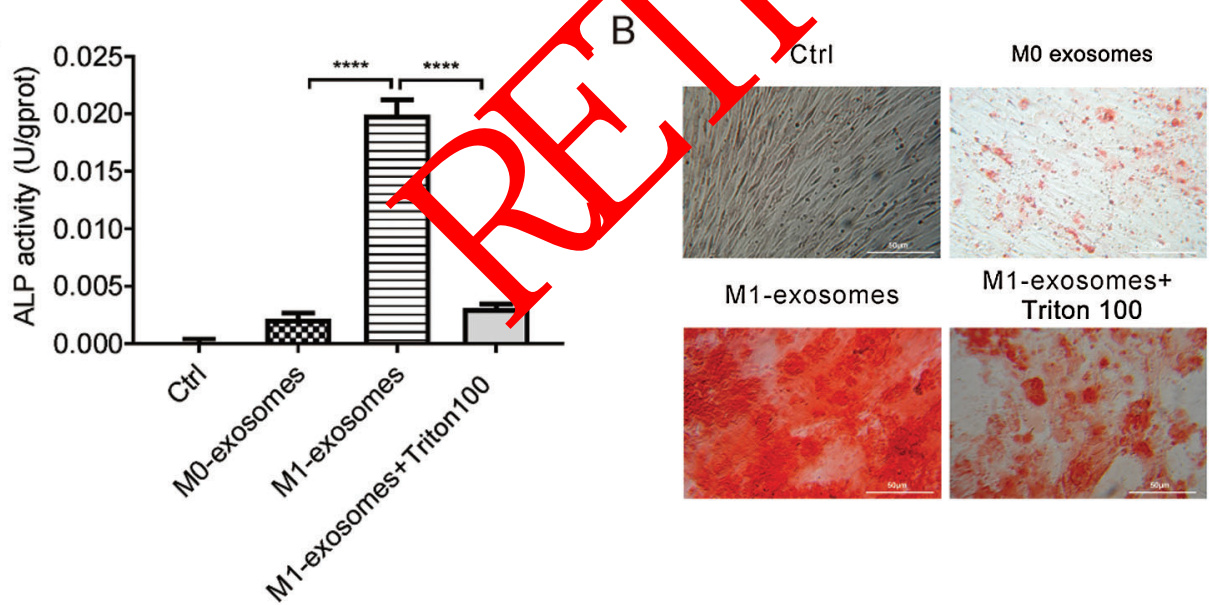

C
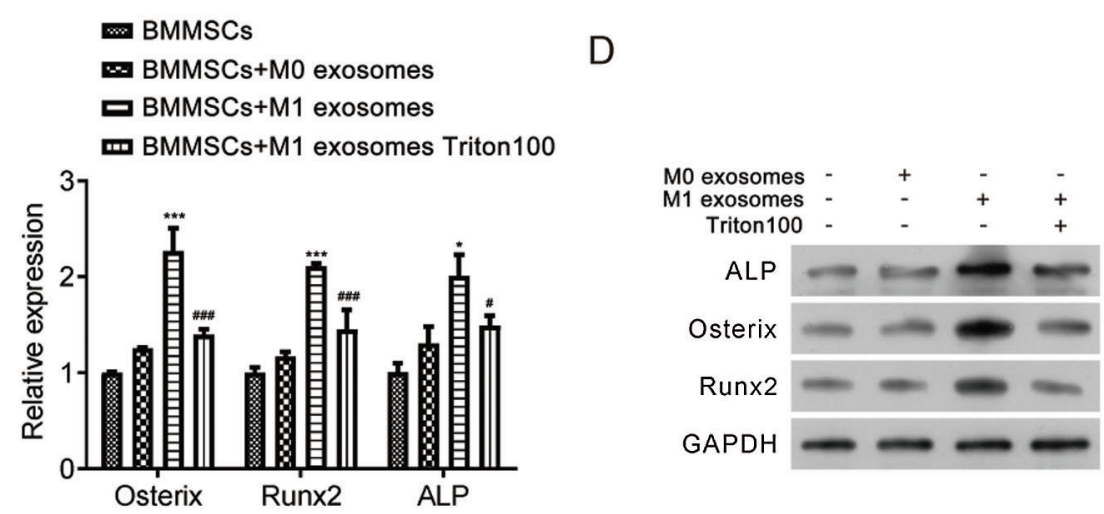

Akt, we examined whether M1-derived exosomes participate in the Akt signaling pathway and regulate BMP2 during BMMSCs differentiation. To experimentally verify this hypothesis, we examined the phosphorylation of Akt and BMP2 expression levels during the differentiation of BMMSCs to examine downstream signaling pathways in the osteogenesis process. Our data showed that M1-derived exosomes activated Akt (phosphorylation level) as shown in Suppl. Fig. 2 and increased the expression of BMP2 (Figs. $4 \mathrm{~A}$ and $4 \mathrm{~B})$. In conclusion, these findings suggest that M1 macrophage derived exosomes may promote the differentiation of BMMSCs through the Akt-BMP2 pathway.

\section{Discussion}

Macrophages have been proved to play an important role in host defending and regulate a range of physiological and pathological events (Krzyszczyk et al., 2018; Li et al., 2019). Macrophages ca participate to regulate the microenvironmen through differentiating into functional cells (Murray ad W n, 20).

Macro ages are a type that can be differentiated into a cies effecte subtypes based on local cells and secre ea onals. all know, macrophages play an active $r$ outwory $l_{e}$ in the process of fracture healing. However, he basic necranism of this beneficial effect is still unclear. order fully explore the role of M1 macrophages in no mation and explore the underlying underlying necrunisms, we focus here on the effect of M1-Exos on
FIGURE 3. Effects of macrophage derived exosomes on the osteogenic differentiation of BMMSCs. (A) Statistical analysis of the ALP activity of M0 or M1-derived exosome-treated BMMSCs. (B) Alizarin Red S staining of M0 or M1-derived exosome-treated BMMSCs. (C-D) Expression levels of osteogenesis-related genes (ALP, Osterix and Runx2) in M0 or M1derived exosomes treated BMMSCs. ${ }^{\star} P<0.05,{ }^{* * *} P<0.001$ vs. $\mathrm{M} 0$ exosomes; ${ }^{\#} P<0.05,{ }^{\# \# \#} P<0.005 v s$. M1 exosomes; Ctrl, control; M0 unpolarized macrophages; M1, polarized macrophages. 


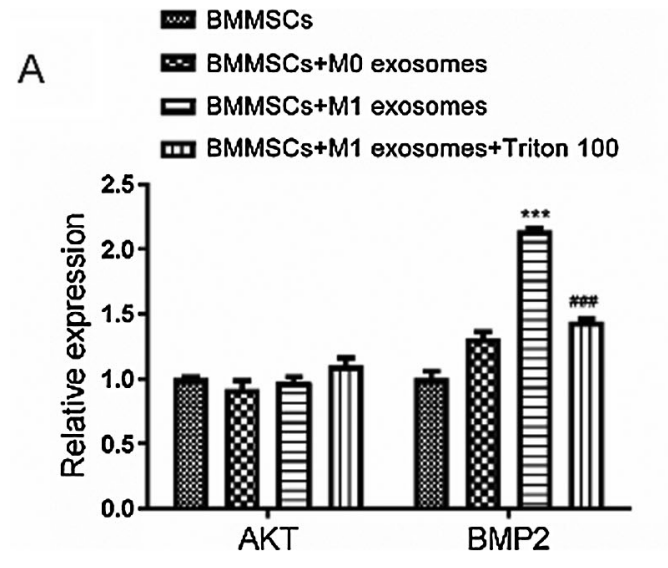

Exosomes are small vesicles that are secreted into circulation by a range of cell types in vivo, whereupon they can be internalized by proximal or distal cells. The small molecules within these exosomes can then regulate the functionality of recipient cells upon internalization, and thereby conducting a communication among various cells and organs. In this study, we found that exosomes derived from $\mathrm{M} 1$ can promote the osteogenic differentiation of BMMSCs and may be involved in the regulation of AKT/RUNX2.

In recently published works, macrophage-derived exosomes have been revealed to regulate many physiological processes (Pajarinen et al., 2019; Saha et al., 2016; Ses et al., 2015). For example, M1-derived exosom important for intestinal stem cell proliferation and se renewal, subsequently maintaining intestinal ymo asis (Saha et al., 2016). Moreover, it has beer eported that exosomes derived from M1 macropb ges n induce endothelial cell proliferation and mi, ation to a celerate wound repair ( $\mathrm{Hu}$ et al., 2018; Li et 201 ). However, the regulation of BMMSCs properti by exiy exosomes remains unclear. Thus, exosome wer from M0 or M1 macrophages. Different metho s, including TEM and NTA staining, were used to iden fy these exosomes. Subsequently, we found that exosomes secreted by different types of macrophages had different effects on BMMSCs differentiation. The Alizarin red S staining and osteogenic gene expression results revealed that only M1-derived exosomes markedly induced the differentiation of BMMSCs. In contrast, M0 derived exosomes exhibited little effect on osteogenic differentiation. This result revealed that only exosomes derived from polarized M1 macrophages influenced the differentiation of BMMSCs. This observation indicates that exosomes not only mediate the microenvironment during pathophysiological processes, but also play a key role during tissue repair, such as during BMMSCs differentiation. In general, our current data indicate that M1-derived exosomes may be used as therapeutic agents to improve the properties of BMMSCs in the regenerative microenvironment.

However, the precise mechanism by which polarized macrophages affect osteogenesis is unclear. In the current study, we mechanistically demonstrated that M1-derived exosomes regulate the Akt signaling pathway, which is critical in osteogenic commitment (Xu et al., 2019; Yu et al., 2016). Moreover, our results suggest that BMP2 is a metabolically regulated substrate of M1-derived exosomes and that this re alation occurs mainly through Akt phosphorylation is is the first report to show a direct relationship tween acror lages and Akt mediated BMP2 function in ost genes Furthermore, to examine the functio al rela nnshir between M1-derived exosomes and $\mathrm{BMP} \angle$ in the diffe stiation of BMMSCs, distinctive models cre used this study. These findings correlate with the ell thou ht hypothesis that M1-derived exosomes activate and hcrease BMP2 levels in osteogenesis, which is nemur for maintaining bone health. In addition, it has Aso veen confirmed that BMP2 is a target of M1-derived exosomes and may act as a regulatory connection oetween macrophages and osteoblast development. Our macrophage differentiation studies revealed that the M1 microenvironment plays a key role in osteogenesis. Although the mRNA levels of BMP2 were increased during osteogenesis, it should also be carefully considered that M1derived exosomes mediate Akt activation and may maintain the homeostasis of osteocytes by stabilizing the BMP2 protein. This finding was evident from the fact that there was persistent Akt activity at all times, as seen in our study. However, there may be other regulatory pathways at the translational or posttranslational level. We will further elucidate the regulatory mechanism between M1-derived exosomes and osteogenesis.

Availability of Data and Materials: The data used and/or analyzed during the current study are available from the corresponding author on reasonable request.

Author Contributions: (I) Conception and design: CGD; (II) Administrative support: CGD; (III) Provision of study materials or patients: TLW, XZ, CHY, WCL; (IV) Collection and assembly of data: TLW, XZ, HTL, YZW; (V) Data analysis and interpretation: ZKK, ZJH, JZL, HRT; (VI) Manuscript writing: CGD, TLW, XZ; (VII) Final approval of manuscript: All authors.

Ethics Approval: All operation on mice were pre-approved by the Animal Care and Use Committee of the Shenzhen University General Hospital (S2019-101607). 
Funding Statement: This work is financially supported by Shenzhen Fundamental Research Funds (No. JCYJ20180305124242438); National Natural Science Foundation of China (81970761); Shenzhen Major Project of Fundamental research (No. JCYJ20200109114233670); Shenzhen Medical and Health Sanming Project (SZSM201911011); Natural Science Foundation of Shenzhen University General Hospital (Nos. SUGH2018QD019, SUGH2018QD014).

Conflicts of Interest: The authors declare that they have no conflicts of interest to report regarding the present study.

\section{References}

Chava S, Chennakesavulu S, Gayatri BM, Reddy ABM (2018). A novel phosphorylation by AMP-activated kinase regulates RUNX2 from ubiquitination in osteogenesis over adipogenesis. Cell Death \& Disease 9: 754.

Ekstrom K, Omar O, Graneli C, Wang X, Vazirisani F, Thomsen P (2013). Monocyte exosomes stimulate the osteogenic gene expression of mesenchymal stem cells. PLoS One 8: e75227.

Fahy N, De Vries-Van Melle ML, Lehmann J, Wei W, Grotenhuis N, Farrell E, Van der Kraan PM, Murphy JM, BastiaansenJenniskens YM, Van Osch GJVM (2014). Human osteoarthritic synovium impacts chondrogenic differentiation of mesenchymal stem cells via macrophage polarisation state. Osteoarthritis Cartilage 22: 1167-1175.

Hu T, Xu H, Wang C, Qin H, An Z (2018). Magnesium enhances the chondrogenic differentiation of mesenchymal stem cells br inhibiting activated macrophage-induced inflammatio Scientific Reports 8: 3406.

Ikebuchi Y, Aoki S, Honma M, Hayashi M, Sugamori Y e dl (201) Coupling of bone resorption and formatio by reverse signalling. Nature 561: 195-200.

Kalluri R, Lebleu VS (2020). The biology, func on, a biomednal applications of exosomes. Science 36.6478.

Kim DY, Kim EJ, Jang WG (2018). Pin ine Aduces osteoblast differentiation through $A \mathrm{Ml}$ pende Runx2 expression. Biochemical and miop, cal Research Communications 495: 1497

Krzyszczyk P, Schloss R, Palmer A, Berthta me F (2018). The role of macrophages in acute and chronic wound healing and interventions to promote pro-wound healing phenotypes. Frontiers in Physiology 9: 419.

Lee J, Kim H, Heo Y, Yoo YK, Han SI et al. (2019). Enhanced paperbased ELISA for simultaneous EVs/exosome isolation and detection using streptavidin agarose-based immobilization. Analyst 145: 157-164.

Li M, Wang T, Tian H, Wei G, Zhao L, Shi Y (2019). Macrophagederived exosomes accelerate wound healing through their anti-inflammation effects in a diabetic rat model. Artificial Cells, Nanomedicine, and Biotechnology 47: 3793-3803.

Ma QL, Fang L, Jiang N, Zhang L, Wang Y et al. (2018). Bone mesenchymal stem cell secretion of sRANKL/OPG/M-CSF in response to macrophage-mediated inflammatory response influences osteogenesis on nanostructured $\mathrm{Ti}$ surfaces. Biomaterials 154: 234-247.

Maxson S, Lopez EA, Yoo D, Danilkovitch-Miagkova A, Leroux MA (2012). Concise review: Role of mesenchymal stem cells in wound repair. Stem Cells Translational Medicine 1: $142-149$.

Mcdonald MK, Tian Y, Qureshi RA, Gormley M, Ertel A et al. (2014). Functional significance of macrophage-derived exosomes in inflammation and pain. Pain 155: 1527-1539.

Murray PJ, Wynn TA (2011). Protective and pathogenic functions of macrophage subsets. Nature Reviews Immunology 11: 723737.

Omar OM, Graneli C, Ekstrom K, Karlsson C, Johansson A et al. (2011). The stimulation of an osteogenic response by classical monocyte activation. Biomaterials 32: 8190-8204.

Pajarinen J, Lin T, Gibon E, Kohno Y, Maruyama M et al. (2019). Mesenchymal stem cell-macrophage crosstalk and bone healing. Biomaterials 196: 80-89.

Poltavtseva RA, Poltavtsev AV, Lutsenko GV, Svirshchevskaya EV (2019). Myths, reality and future of mesenchymal stem cell therapy. Cell and Tissue Research 375: 563-574.

Regmi S, Pathak S, Kim JO, Yong CS, Jeong JH (2019). Mesenchymal stem cell therapy for the treatment of inflammatory diseases: Challenges, op r atunities, and future perspectives. European Journal of $C$ Biology 98: 151041.

Saha S, Aranda $C, \mathrm{H}_{a}$, kawa Bhanja P, Atay S et al. (2016). Macr shage-deriv e racellular vesicle-packaged WNTs recue th otinal stom cells and enhance survival after diation ury ature Communications 7: 13096.

Sesias B, D $\mathrm{r}$ R, Meu_rros da Cunha C, Todorov A, Schaeren S et al. (2015). Anti-inflammatory/tissue repair macrophages enk nce the cartilage-forming capacity of human bone mal ow-derived mesenchymal stromal cells. Journal of ular Physiology 230: 1258-1269.

G, Kumar Y, Khan MP, Shukla N, Kapoor I et al. (2016). Skp2 inhibits osteogenesis by promoting ubiquitinproteasome degradation of Runx2. Biochimica et Biophysica Acta 1863: 510-509.

Wei F, Li M, Crawford R, Zhou Y, Xiao Y (2019). Exosomeintegrated titanium oxide nanotubes for targeted bone regeneration. Acta Biomaterialia 86: 480-492.

Wei X, Yang X, Han ZP, Qu FF, Shao L, Shi YF (2013). Mesenchymal stem cells: A new trend for cell therapy. Acta Pharmacologica Sinica 34: 747-754.

Xu XY, He XT, Wang J, Li X, Xia Y et al. (2019). Role of the P2X7 receptor in inflammation-mediated changes in the osteogenesis of periodontal ligament stem cells. Cell Death \& Disease 10: 20.

Yu B, Sondag GR, Malcuit C, Kim MH, Safadi FF (2016). Macrophage-associated osteoactivin/GPNMB mediates mesenchymal stem cell survival, proliferation, and migration via a CD44-dependent mechanism. Journal of Cellular Biochemistry 117: 1511-1521.

Zhang Q, Hwang JW, Oh JH, Park CH, Chung SH et al. (2017a). Effects of the fibrous topography-mediated macrophage phenotype transition on the recruitment of mesenchymal stem cells: An in vivo study. Biomaterials 149: 77-87.

Zhang Y, Bose T, Unger RE, Jansen JA, Kirkpatrick CJ, van den Beucken J (2017b). Macrophage type modulates osteogenic differentiation of adipose tissue MSCs. Cell and Tissue Research 369: 273-286.

Zhang Y, Liu Y, Liu H, Tang WH (2019). Exosomes: Biogenesis, biologic function and clinical potential. Cell \& Bioscience 9: 19. 


\section{Appendix}

A

Alizarin Red staining
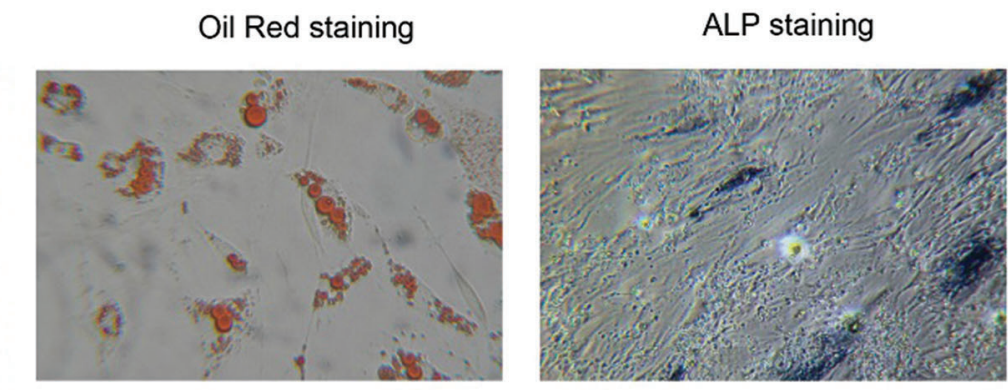

$\mathbf{B}$
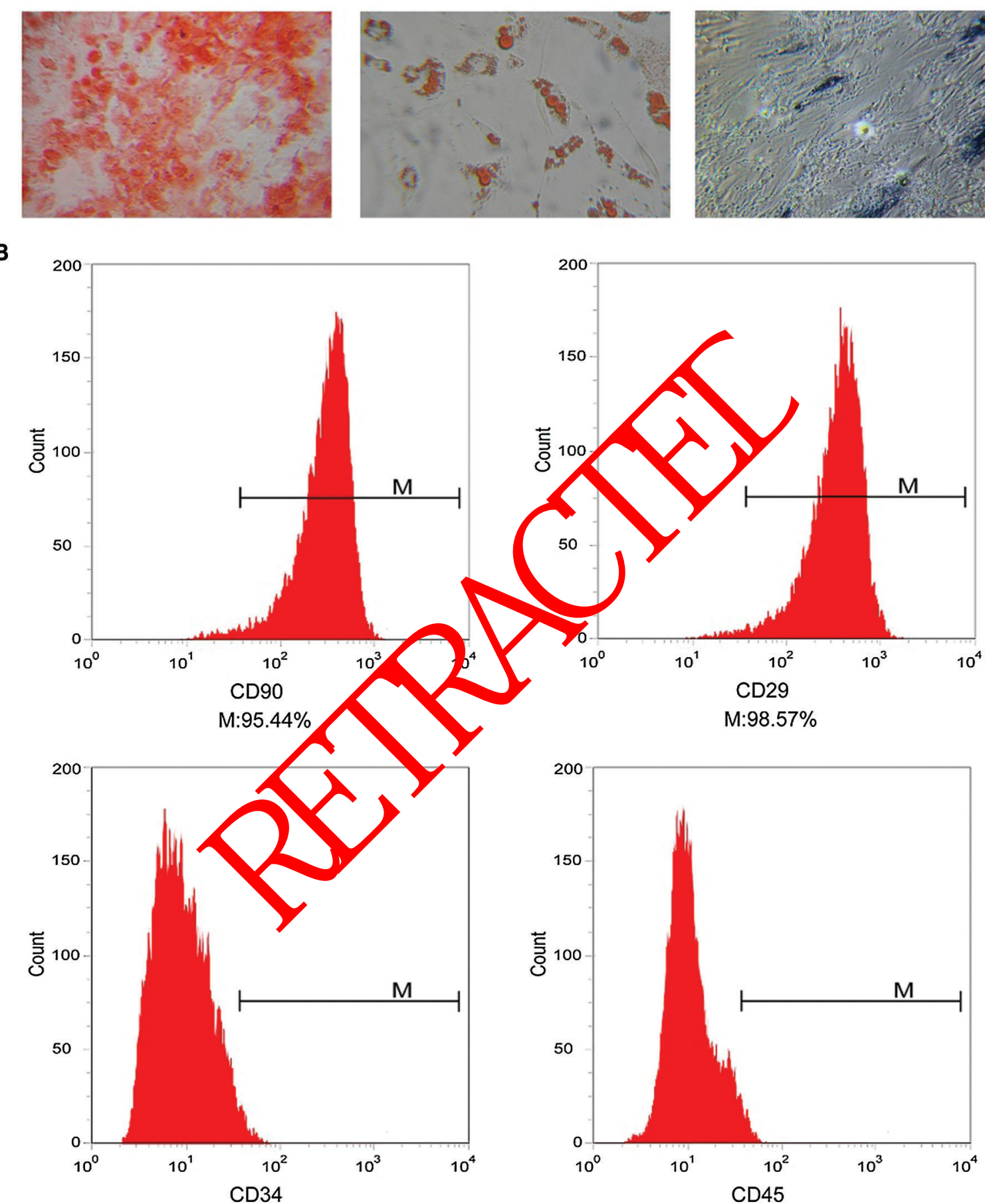

M:3.66\%

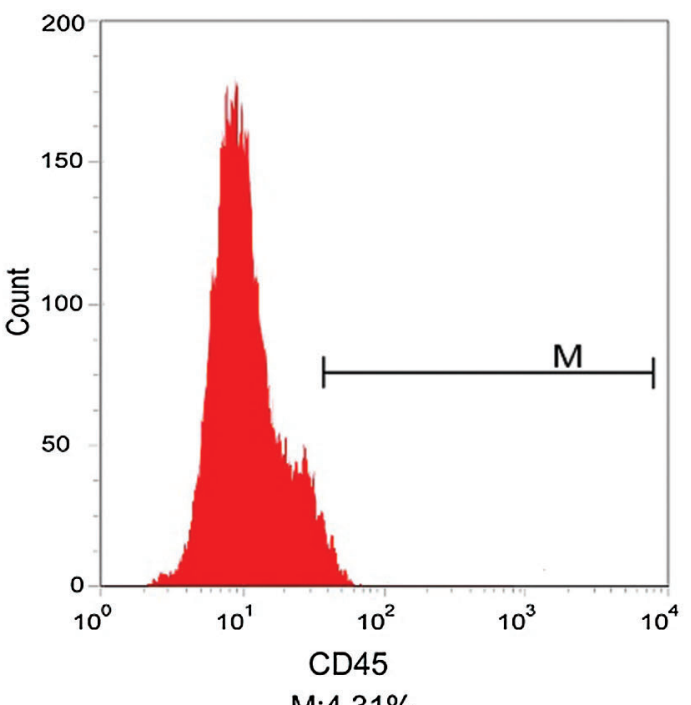

SUPPLEMENTARY FIGURE 1. BMMSCs cell identification. (A) The results of Alizarin Red Oil Red O and ALP staining. (B) Identification of BMMSCs by flow cytometry. 
A

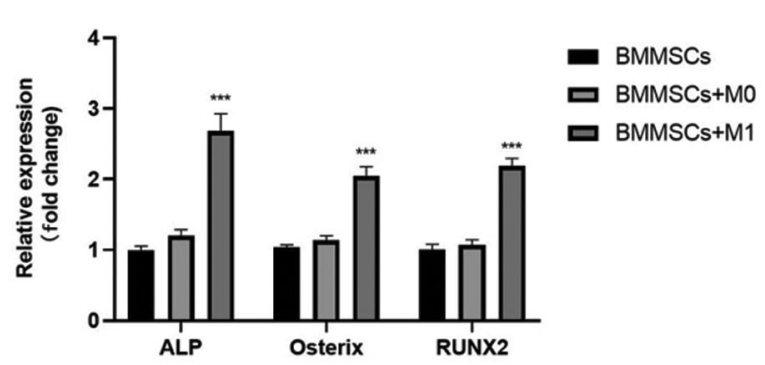

B

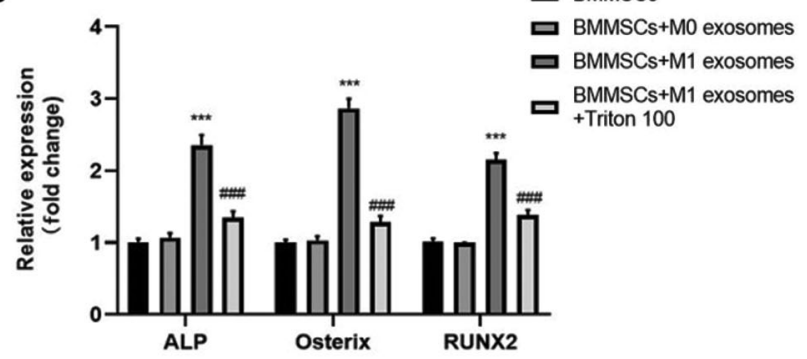

C

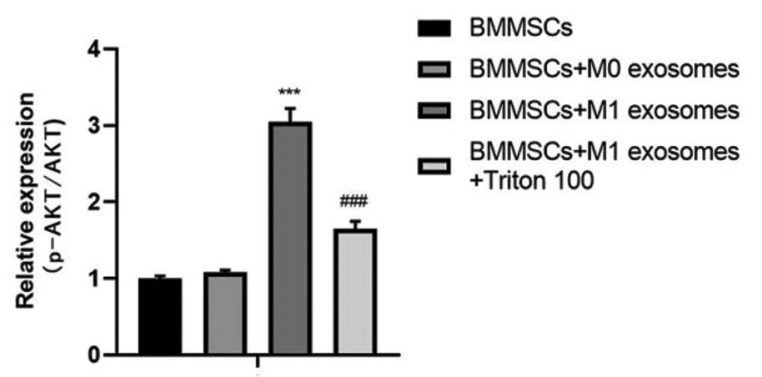

D

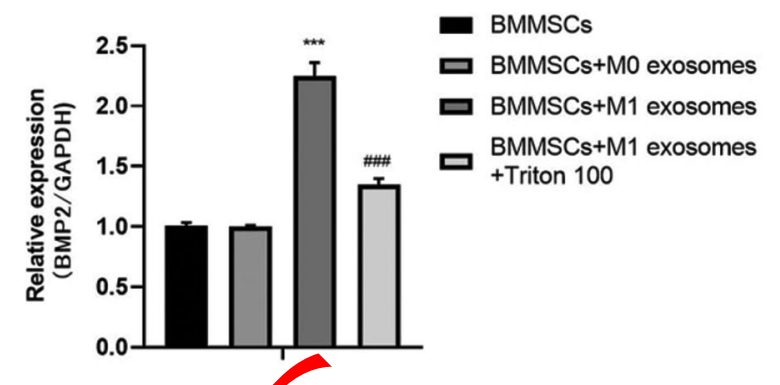

SUPPLEMENTARY FIGURE 2. The quantitative analysis histogram of western blot. ${ }^{* *} P<.005 ;{ }^{\# \# *} P<0.005$. 